\title{
Corporate Social Responsibility and Lawsuit Settlement: Evidence from Special Items
}

Christopher J. Skousen

Utah State University

\section{Li Sun}

The University of Tulsa

huntsman.usu.edu 


\section{Corporate Social Responsibility and Lawsuit Settlement: Evidence from Special Items}

\section{$\underline{\text { Outline }}$}

- Background-CSR

- Motivation

- Introduction

- Literature Review and Hypotheses Development

- Research Design

- Sample Selection and Descriptive Statistics

- Main Tests

- Additional Tests

- Conclusion 


\section{Background - CSR}

- Corporate social responsibility (CSR) has become an important research topic in business (e.g., Malik, 2005).

- Definition of CSR - integration of social practices such as environmental, ethical, and legal practices into a company's business model.

- The number of CSR studies has been increasing in accounting research (Moser and Martin, 2012). 


\section{Background - CSR}

One critical theory of CSR is known as the stakeholder view/theory.

1. Money is not everything. Shareholders are not the only stakeholders.

2. Companies must care for other stakeholders such as suppliers, customers, employees, and other parties.

3. If companies try to satisfy the needs of all stakeholders, the stakeholders will support such firms (i.e., CSR firms) as a return.

4. Thus, under the stakeholder view, high CSR firms often receive benefits and favorable outcomes from their stakeholders. 


\section{Motivation}

1. JUUL Lawsuit in 2019

- Bad products (harmful chemicals to human lungs) -> irresponsible CSR

○ False marketing (safer and better than smoking) -> irresponsible CSR

- The first lawsuit filed on October 16, 2019; JUUL is in process of settling lawsuits.

2. General expectations and anecdotal evidence -> good companies are less likely to be sued.

3. Any empirical evidence on the legal outcomes of CSR in accounting research? 


\section{Introduction}

- The purpose of this study is to examine the impact of CSR on corporate lawsuit characteristics (i.e., the probability of lawsuit and the magnitude of lawsuit settlement).

- We rely on the account of lawsuit settlement (SETP, Compustat Item \#372) in the special items, which is nonrecurring in nature and is reported as a component of other income within income from continuing operations on an income statement.

- A company can either report a settlement gain (i.e., usually the plaintiff in a lawsuit) or a loss (i.e., usually the defendant in a lawsuit). 


\section{Introduction}

In testing $\mathrm{H} 1$ - (CSR and the likelihood of lawsuit)

- Using a large panel sample with 21,761 observations from 1991 to 2016, we find a significant negative relation between CSR performance and the probability of lawsuit, which suggests that socially responsible firms are less likely to be involved in lawsuits.

- We also find that the significant negative relation becomes weaker for firms with more cash, consistent with anecdotal evidence that firms with more money are more likely to be sued. 


\section{Introduction}

In testing $\mathrm{H} 2$ - (CSR and the magnitude of lawsuit settlement)

- Using a panel sample with 4,307 observations reporting settlement gains or losses from 1991 to 2016, we find a significant positive relation between CSR and the magnitude of settlement, which suggests that socially responsible firms are more likely to receive favorable outcomes (i.e., larger settlement gain or smaller settlement loss).

- We find that the significant relation between CSR and the settlement size is largely driven by firms with settlement loss (i.e., firms being sued). 


\section{Contributions}

- This study provides empirical evidence that not only supports the stakeholder view of CSR but is also consistent with anecdotal evidence and general expectations.

- This study not only answers the call in Johnson, Lopez and Sanchez (2011) but also raises new research questions about special items.

- Practical implications

- If managers are risk averse, engaging in CSR activities can effectively mitigate legal risks;

- CSR firms receive favorable settlement outcomes - larger(smaller) settlement gain(loss).

- Lowering the level of corporate cash holdings might help reduce legal risks. 


\section{Literature Review on CSR}

- The first category examines the impact of CSR activities on firm performance and outcomes.

- The association between CSR performance and financial performance of a firm

- The impact of CSR on other variables

- The second category investigates factors that can influence a firm's CSR activities and performance. 


\section{Literature Review}

The first category

The association between CSR performance and financial performance

- A large body of prior research documents a significant positive association between social performance and financial performance of a firm (e.g., McGuire, Sundgren, and Schnessweis, 1988; Smith, 1994; Porter and Van der Linde, 1995; Waddock and Graves, 1997; Berman, Wicks, Kotha, and Jones, 1999; Margolis and Walsh, 2003; Smith, 2003; Orlitzky, Schmidt, and Rynes, 2003; Carmeli, Gilat, and Waldman, 2007; Beurden and Gossling, 2008; Brammer and Millington, 2008; and many others).

- win-win 


\section{Literature Review}

The first category

The impact of CSR performance on other variables

- CSR can increase firm value (e.g., Jo and Harjoto, 2011; Deng, Kang, and Low. 2013; Malik, 2015), improve bond credit ratings (e.g., Attig, Ghoul, Guedhami, and Suh, 2013; Jiraporn, Jiraporn, Boeprasert, and Chang, 2014), increase the value and the level of cash holdings (e.g., Arouri and Pijourlet, 2017; Cheung, 2016), improve the quality of earnings (e.g., Kim et al., 2012), reduce information asymmetry (e.g., Cho, Lee, and Pfeiffer, 2013), constrain tax avoidance activities (e.g., Hoi, Wu, and Zhang, 2013; Lanis and Richardson, 2015), reduce firm risks (e.g., Godfrey, Merrill, and Hansen, 2009), reduce cost of capital (e.g., Dhaliwal, Li, Tsang, and Yang, 2011; Dhaliwal et al., 2014), increase analyst forecast accuracy (e.g., Dhaliwal, Radhakrishnan, Tsang, and Yang, 2012), influence seasoned equity offerings (e.g., Dutordoir, Strong, and Sun, 2018), lower debt financing costs (e.g., Ye and Zhang, 2011), and reduce stock price crash risks (e.g., Kim et al, 2014).

- This stream suggests that CSR firms can bring benefits. 


\section{Literature Review}

The first category

The impact of CSR performance on other variables

- Christensen (2016) is closely related to our study. He examines and finds that companies reporting their accountability activities (e.g., CSR activities) are less likely to engage in misconducts such as bribery and kickbacks. Our study is different from Christensen (2016) as we focus on CSR performance.

- Our study belongs to the CSR Performance stream. 


\section{Literature Review}

The second category

Factors that influence the level of CSR

A few recent studies

- Chen, Zhou, and Zhu (2018) find that CEOs with shorter (longer) tenure engage in more (fewer) CSR activities.

- Bouslah, Linares-Zegarra, M'Zali, and Scholtens (2018) find that risktaking CEOs are more likely to engage in irresponsible CSR activities.

- Sun, Walkup, and Wu (2019) find that firms with high sales order backlog engage in more irresponsible CSR activities. 


\section{Hypotheses Development}

- Moser and Martin (2012) encourage accounting researchers to adopt the stakeholder view of CSR.

- Doing good things for stakeholders -> good relationships with stakeholders

- More ethical - do the right things -> lower legal risks

- CSR firms often receive benefits and favorable outcomes.

H1: CSR firms are less likely to be involved in lawsuits. H2: CSR firms are more likely to receive favorable lawsuit settlement outcomes. 


\section{Research Design}

Measuring CSR performance

- Following the large body of prior research, we use the CSR ratings from the MSCI's ESG database. [7 CSR dimensions]

\begin{tabular}{l|c|c|c|}
\multicolumn{1}{c}{ CSR Components } & \multicolumn{3}{c|}{ Example (Company XYZ) } \\
1. Community Relations & 9 & 8 & Net \\
2. Corporate Governance & 7 & 6 & 1 \\
3. Diversity & 5 & 4 & 1 \\
4. Employee Relations & 3 & 2 & 1 \\
5. Environment & 1 & 0 & 1 \\
6. Human Rights & 0 & 1 & -1 \\
7. Product & 0 & 2 & -2 \\
& & TOTAL CSR $=$ & $\mathbf{2}$
\end{tabular}




\section{Research Design}

Measuring CSR performance

- $\mathrm{CSR}=($ Total strengths of Community Relations - Total concerns of Community Relations) + (Total strengths of Corporate Governance - Total concerns of Corporate Governance) + (Total strengths of Diversity - Total concerns of Diversity) + (Total strengths of Employee Relations - Total concerns of Employee Relations) + (Total strengths of Environment - Total concerns of Environment) + (Total strengths of Human Rights - Total concerns of Human Rights) + (Total strengths of Product - Total concerns of Product)

- CSR_STRENGTH = Total strengths of Community Relations + Total strengths of Corporate Governance + Total strengths of Diversity + Total strengths of Employee Relations + Total strengths of Environment + Total strengths of Human Rights + Total strengths of Product

- CSR_CONCERN = Total concerns of Community Relations + Total concerns of Corporate Governance + Total concerns of Diversity + Total concerns of Employee Relations + Total concerns of Environment + Total concerns of Human Rights + Total concerns of Product 


\section{Research Design}

\section{Measuring Lawsuit Settlement}

- In the section of special items, companies report lawsuit (or legal) settlement (SETP).

- LAWSUIT - an indicator variable that equals one if a firm is involved in (at least) one lawsuit in a given year.

- LAWSUIT_GL - the magnitude or size of settlement gain or loss, scaled by total firm assets.

An example - Comcast Corporation 2018 10-K report - income statement

Year ended December 31 (in millions) Adjusted EBITDA

Adjustment for legal settlement

Adjustment for Sky transaction-related costs

Depreciation

Amortization

Other operating gains

Interest expense

Investment and other income (loss), net

Income before income taxes

$\begin{array}{rcc}2018 & 2017 & 2016 \\ \$ 30,165 & \$ 27,956 & \$ 26,257 \\ (\mathbf{1 2 5}) & \mathbf{( 2 5 0 )} & - \\ (355) & - & - \\ (8,281) & (7,914) & (7,464) \\ (2,736) & (2,216) & (1,962) \\ 341442 & - & \\ (3,542) & (3,086) & (2,942) \\ (225) & 421 & 437 \\ \$ 15,242 & \$ 15,353 & \$ 14,326\end{array}$




\section{Research Design}

Empirical Specification

- Corporate Lawsuit $_{\mathrm{i}, \mathrm{t}}=\beta_{0}+\beta_{1} \mathbf{C S R}_{\mathrm{i}, \mathrm{t}}+\beta_{2} \mathrm{SIZE}_{\mathrm{i}, \mathrm{t}}+\beta_{3} \mathrm{MTB}_{\mathrm{i}, \mathrm{t}}+\beta_{4} \mathrm{LEV}_{\mathrm{i}, \mathrm{t}}+$ $\beta_{5} \mathrm{ROA}_{\mathrm{i}, \mathrm{t}}+\beta_{6} \mathrm{CASHFL}_{\mathrm{i}, \mathrm{t}}+\beta_{7} \mathrm{LOSS}_{\mathrm{i}, \mathrm{t}}+\beta_{8} \mathrm{ZSCORE}_{\mathrm{i}, \mathrm{t}}+\beta_{9} \mathrm{TOBINQ}_{\mathrm{i}, \mathrm{t}}+$ $\beta_{10}$ FIRMAGE $_{\mathrm{i}, \mathrm{t}}+\beta_{11}$ ASSETAGE $_{\mathrm{i}, \mathrm{t}}+\beta_{12}$ MGR_ABILITY $_{\mathrm{i}, \mathrm{t}}+$ $\beta_{13}$ ACCRUAL $_{i, t}+\beta_{14}$ SALE_VOL $_{i, t}+\beta_{15}$ SPE_ITEM $_{i, t}+\beta_{16}$ BIG4 $_{i, t}+$ $\beta_{17} \mathrm{HIGH} \_\mathrm{RISK}_{\mathrm{i}, \mathrm{t}}+$ Industry Indicators + Year Indicators $+\varepsilon_{\mathrm{i}, \mathrm{t}}$ 


\section{Research Design}

\section{Empirical Specification - control variables}

$\begin{array}{ll}\text { SIZE } & \text { total assets } \\ \text { MTB } & \text { market to book ratio } \\ \text { LEV } & \text { leverage ratio } \\ \text { ROA } & \text { return on assets } \\ \text { CASHFL } & \text { operating cash flos } \\ \text { LOSS } & \text { loss indicator } \\ \text { ZSCORE } & \text { altman Z score } \\ \text { TOBINQ } & \text { tobin's q } \\ \text { FIRMAGE } & \text { firm age } \\ \text { ASSETAGE } & \text { asset age } \\ \text { MGR_ABILITY } & \text { managerial ability } \\ \text { ACCRUAL } & \text { discretionary accrual } \\ \text { SALE_VOL } & \text { sales volatility } \\ \text { SPE_ITEM } & \text { special items } \\ \text { BIG4 } & \text { BIG4 auditor } \\ \text { HIGH_RISK } & \text { industries with high litigation risk }\end{array}$


Sample Selection and Descriptive Statistics (Table 1)

\begin{tabular}{|l|c|} 
& Observations \\
\hline CSR data from MSCI's ESG database (1991-2016) & $\mathbf{5 3 , 1 6 8}$ \\
\hline $\begin{array}{l}\text { Less: Observations with insufficient data to construct } \\
\text { independent variable of interest and control variables }\end{array}$ & $\mathbf{( 2 8 , 2 9 5 )}$ \\
\hline $\begin{array}{l}\text { Less: Observations in highly regulated industries (SIC 4000- } \\
\text { 4999 \& 6000-6999) }\end{array}$ & $\mathbf{( 3 , 1 1 2 )}$ \\
\hline Number of firm-year observations & $\mathbf{2 1 , 7 6 1}$ \\
\hline Number of unique firms & $\mathbf{2 , 4 3 7}$ \\
\hline
\end{tabular}


Sample Selection and Descriptive Statistics

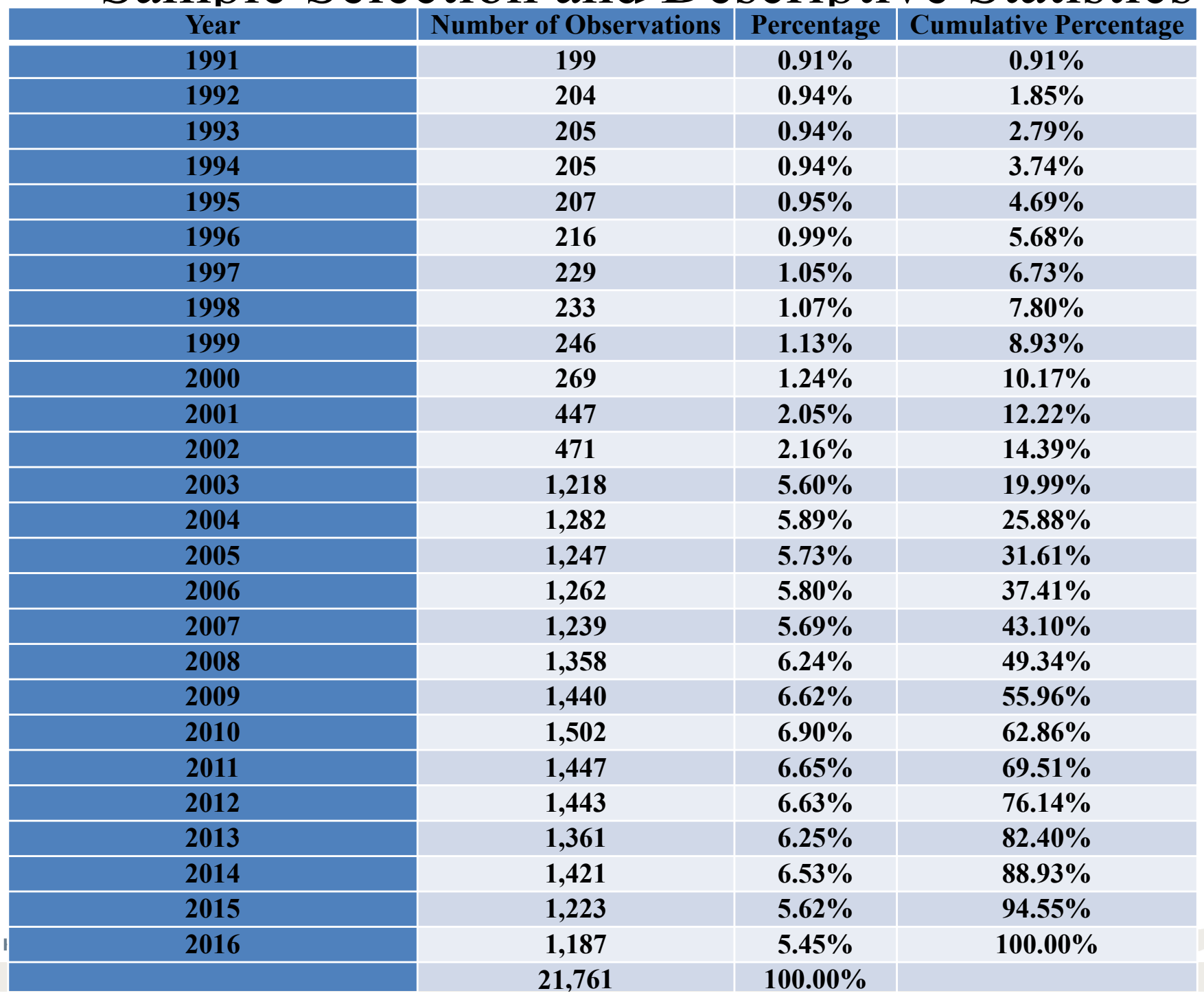




\section{Sample Selection and Descriptive Statistics (Table 2)}

\begin{tabular}{|l|c|c|c|c|c|c|}
\hline Variable & Obs. & Mean & Std Dev & 25 th Pctl & 50th Pctl & 75 th Pctl \\
\hline LAWSUIT & 21,761 & $\mathbf{0 . 1 9 8}$ & $\mathbf{0 . 3 9 8}$ & $\mathbf{0 . 0 0 0}$ & $\mathbf{0 . 0 0 0}$ & $\mathbf{0 . 0 0 0}$ \\
\hline CSR & 21,761 & $\mathbf{- 0 . 0 1 9}$ & $\mathbf{2 . 5 4 4}$ & $\mathbf{- 1 . 0 0 0}$ & $\mathbf{0 . 0 0 0}$ & $\mathbf{1 . 0 0 0}$ \\
\hline SIZE & 21,761 & $\mathbf{7 . 2 0 1}$ & $\mathbf{1 . 5 7 9}$ & $\mathbf{6 . 0 2 5}$ & $\mathbf{7 . 1 0 1}$ & $\mathbf{8 . 2 8 0}$ \\
\hline MTB & 21,761 & $\mathbf{3 . 4 2 6}$ & $\mathbf{5 . 0 2 5}$ & $\mathbf{1 . 5 8 6}$ & $\mathbf{2 . 5 0 2}$ & $\mathbf{4 . 0 1 8}$ \\
\hline LEV & 21,761 & $\mathbf{0 . 1 7 8}$ & $\mathbf{0 . 1 7 8}$ & $\mathbf{0 . 0 0 5}$ & $\mathbf{0 . 1 4 7}$ & $\mathbf{0 . 2 7 8}$ \\
\hline ROA & 21,761 & $\mathbf{0 . 0 3 3}$ & $\mathbf{0 . 1 2 6}$ & $\mathbf{0 . 0 1 4}$ & $\mathbf{0 . 0 5 2}$ & $\mathbf{0 . 0 9 0}$ \\
\hline CASHFL & 21,761 & $\mathbf{0 . 0 9 6}$ & $\mathbf{0 . 1 0 3}$ & $\mathbf{0 . 0 5 9}$ & $\mathbf{0 . 1 0 1}$ & $\mathbf{0 . 1 4 8}$ \\
\hline LOSS & 21,761 & $\mathbf{0 . 2 0 3}$ & $\mathbf{0 . 4 0 2}$ & $\mathbf{0 . 0 0 0}$ & $\mathbf{0 . 0 0 0}$ & $\mathbf{0 . 0 0 0}$ \\
\hline ZSCORE & 21,761 & $\mathbf{4 . 8 5 0}$ & $\mathbf{5 . 0 8 4}$ & $\mathbf{2 . 2 5 9}$ & $\mathbf{3 . 6 2 4}$ & $\mathbf{5 . 7 8 5}$ \\
\hline TOBINQ & 21,761 & $\mathbf{2 . 1 7 3}$ & $\mathbf{1 . 4 0 4}$ & $\mathbf{1 . 3 0 4}$ & $\mathbf{1 . 7 3 2}$ & $\mathbf{2 . 5 0 4}$ \\
\hline FIRMAGE & 21,761 & $\mathbf{3 . 1 4 0}$ & $\mathbf{0 . 6 1 7}$ & $\mathbf{2 . 6 3 9}$ & $\mathbf{3 . 0 9 1}$ & $\mathbf{3 . 7 1 4}$ \\
\hline ASSETAGE & 21,761 & $\mathbf{0 . 4 7 8}$ & $\mathbf{0 . 1 5 3}$ & $\mathbf{0 . 3 7 3}$ & $\mathbf{0 . 4 7 0}$ & $\mathbf{0 . 5 8 0}$ \\
\hline MGR_ABLITY & 21,761 & $\mathbf{0 . 5 6 0}$ & $\mathbf{0 . 2 9 6}$ & $\mathbf{0 . 3 0 0}$ & $\mathbf{0 . 6 0 0}$ & $\mathbf{0 . 8 0 0}$ \\
\hline ACCRUAL & 21,761 & $\mathbf{0 . 0 5 7}$ & $\mathbf{0 . 0 5 7}$ & $\mathbf{0 . 0 1 8}$ & $\mathbf{0 . 0 4 1}$ & $\mathbf{0 . 0 7 5}$ \\
\hline SALE_VOL & 21,761 & $\mathbf{0 . 7 4 4}$ & $\mathbf{0 . 5 4 6}$ & $\mathbf{0 . 3 5 6}$ & $\mathbf{0 . 6 0 2}$ & $\mathbf{0 . 9 7 4}$ \\
\hline SPE_ITEM & 21,761 & $\mathbf{- 0 . 0 1 5}$ & $\mathbf{0 . 0 5 0}$ & $\mathbf{- 0 . 0 1 3}$ & $\mathbf{- 0 . 0 0 2}$ & $\mathbf{0 . 0 0 0}$ \\
\hline BIG4 & 21,761 & $\mathbf{0 . 9 0 5}$ & $\mathbf{0 . 2 9 3}$ & $\mathbf{1 . 0 0 0}$ & $\mathbf{1 . 0 0 0}$ & $\mathbf{1 . 0 0 0}$ \\
\hline HIGH_RISK & 21,761 & $\mathbf{0 . 2 5 0}$ & $\mathbf{0 . 4 3 3}$ & $\mathbf{0 . 0 0 0}$ & $\mathbf{0 . 0 0 0}$ & $\mathbf{0 . 0 0 0}$ \\
\hline
\end{tabular}




\section{Sample Selection and Descriptive Statistics}

\begin{tabular}{l}
\hline Variable \\
\hline CSR \\
\hline SIZE \\
\hline MTB \\
\hline LEV \\
\hline ROA \\
\hline CASHFL \\
\hline LOSS \\
\hline ZSCORE \\
\hline TOBINQ \\
\hline FIRMAGE \\
\hline ASSETAGE \\
\hline MGR_ABLITY \\
\hline ACCRUAL \\
\hline SALE_VOL \\
\hline SPE_ITEM \\
\hline BIG4 \\
\hline HIGH_RISK \\
\hline
\end{tabular}

\begin{tabular}{|c|c|c|c|c|c|}
\hline \multicolumn{2}{|c|}{ LAWSUIT = 1} & \multicolumn{2}{|c|}{ LAWSUIT $=0$} & \multirow[b]{2}{*}{ Diff. in Mean } & \multirow[b]{2}{*}{ p-value } \\
\hline Obs. & Mean & Obs. & Mean & & \\
\hline 4,307 & -0.108 & 17,454 & 0.003 & $-0.111 * * *$ & 0.001 \\
\hline 4,307 & 7.610 & 17,454 & 7.100 & $0.510 * * *$ & $<0.0001$ \\
\hline 4,307 & 3.170 & 17,454 & 3.489 & $-0.319 * * *$ & 0.000 \\
\hline 4,307 & 0.196 & 17,454 & 0.174 & $0.022 * * *$ & $<0.0001$ \\
\hline 4,307 & 0.032 & 17,454 & 0.033 & -0.001 & 0.592 \\
\hline 4,307 & 0.095 & 17,454 & 0.096 & -0.001 & 0.545 \\
\hline 4,307 & 0.213 & 17,454 & 0.201 & $0.012 *$ & 0.070 \\
\hline 4,307 & 4.085 & 17,454 & 5.039 & $-0.954 * * *$ & $<0.0001$ \\
\hline 4,307 & 1.944 & 17,454 & 2.230 & $-0.286 * * *$ & $<0.0001$ \\
\hline 4,307 & 3.224 & 17,454 & 3.119 & $0.105 * * *$ & $<0.0001$ \\
\hline 4,307 & 0.474 & 17,454 & 0.480 & $-0.006 * *$ & 0.029 \\
\hline 4,307 & 0.549 & 17,454 & 0.563 & $-0.014 * * *$ & 0.006 \\
\hline 4,307 & 0.053 & 17,454 & 0.058 & $-0.005 * * *$ & $<0.0001$ \\
\hline 4,307 & 0.674 & 17,454 & 0.761 & $-0.087 * * *$ & $<0.0001$ \\
\hline 4,307 & -0.018 & 17,454 & -0.014 & $-0.004 * * *$ & $<0.0001$ \\
\hline 4,307 & 0.928 & 17,454 & 0.899 & $0.029 * * *$ & $<0.0001$ \\
\hline 4,307 & 0.236 & 17,454 & 0.253 & $-0.017 * *$ & 0.019 \\
\hline
\end{tabular}




\section{Sample Selection and Descriptive Statistics}

\begin{tabular}{|l|c|c|c|c|c|c|}
\hline Variable & Obs. & Mean & Std Dev & 25th Pctl & 50th Pctl & 75th Pctl \\
\hline LAWSUIT_GL & $\mathbf{4 , 3 0 7}$ & $\mathbf{- 0 . 0 0 2}$ & $\mathbf{0 . 0 2 1}$ & $\mathbf{- 0 . 0 0 4}$ & $\mathbf{0 . 0 0 0}$ & $\mathbf{0 . 0 0 3}$ \\
\hline CSR & $\mathbf{4 , 3 0 7}$ & $\mathbf{- 0 . 1 0 8}$ & $\mathbf{2 . 8 6 7}$ & $\mathbf{- 2 . 0 0 0}$ & $\mathbf{0 . 0 0 0}$ & $\mathbf{1 . 0 0 0}$ \\
\hline SIZE & $\mathbf{4 , 3 0 7}$ & $\mathbf{7 . 6 1 7}$ & $\mathbf{1 . 5 6 0}$ & $\mathbf{6 . 4 9 2}$ & $\mathbf{7 . 5 1 0}$ & $\mathbf{8 . 7 2 5}$ \\
\hline MTB & $\mathbf{4 , 3 0 7}$ & $\mathbf{3 . 1 9 8}$ & $\mathbf{7 . 3 4 1}$ & $\mathbf{1 . 5 3 9}$ & $\mathbf{2 . 3 6 0}$ & $\mathbf{3 . 6 8 8}$ \\
\hline LEV & $\mathbf{4 , 3 0 7}$ & $\mathbf{0 . 1 9 6}$ & $\mathbf{0 . 1 7 7}$ & $\mathbf{0 . 0 3 6}$ & $\mathbf{0 . 1 7 2}$ & $\mathbf{0 . 2 9 1}$ \\
\hline ROA & $\mathbf{4 , 3 0 7}$ & $\mathbf{0 . 0 3 2}$ & $\mathbf{0 . 1 0 7}$ & $\mathbf{0 . 0 1 1}$ & $\mathbf{0 . 0 4 7}$ & $\mathbf{0 . 0 8 1}$ \\
\hline CASHFL & $\mathbf{4 , 3 0 7}$ & $\mathbf{0 . 0 9 6}$ & $\mathbf{0 . 0 8 1}$ & $\mathbf{0 . 0 5 6}$ & $\mathbf{0 . 0 9 5}$ & $\mathbf{0 . 1 3 8}$ \\
\hline LOSS & $\mathbf{4 , 3 0 7}$ & $\mathbf{0 . 2 1 3}$ & $\mathbf{0 . 4 0 9}$ & $\mathbf{0 . 0 0 0}$ & $\mathbf{0 . 0 0 0}$ & $\mathbf{0 . 0 0 0}$ \\
\hline ZSCORE & $\mathbf{4 , 3 0 7}$ & $\mathbf{4 . 0 6 5}$ & $\mathbf{4 . 0 5 9}$ & $\mathbf{2 . 0 2 3}$ & $\mathbf{3 . 2 2 2}$ & $\mathbf{4 . 9 5 0}$ \\
\hline TOBINQ & $\mathbf{4 , 3 0 7}$ & $\mathbf{1 . 9 3 8}$ & $\mathbf{1 . 1 1 7}$ & $\mathbf{1 . 2 6 9}$ & $\mathbf{1 . 6 2 1}$ & $\mathbf{2 . 2 0 5}$ \\
\hline FIRMAGE & $\mathbf{4 , 3 0 7}$ & $\mathbf{3 . 2 2 4}$ & $\mathbf{0 . 6 2 7}$ & $\mathbf{2 . 7 0 8}$ & $\mathbf{3 . 1 7 8}$ & $\mathbf{3 . 8 2 9}$ \\
\hline ASSETAGE & $\mathbf{4 , 3 0 7}$ & $\mathbf{0 . 4 7 4}$ & $\mathbf{0 . 1 4 7}$ & $\mathbf{0 . 3 7 1}$ & $\mathbf{0 . 4 6 6}$ & $\mathbf{0 . 5 6 8}$ \\
\hline MGR_ABLITY & $\mathbf{4 , 3 0 7}$ & $\mathbf{0 . 5 4 9}$ & $\mathbf{0 . 2 9 6}$ & $\mathbf{0 . 3 0 0}$ & $\mathbf{0 . 5 0 0}$ & $\mathbf{0 . 8 0 0}$ \\
\hline ACCRUAL & $\mathbf{4 , 3 0 7}$ & $\mathbf{0 . 0 5 3}$ & $\mathbf{0 . 0 5 2}$ & $\mathbf{0 . 0 1 7}$ & $\mathbf{0 . 0 3 9}$ & $\mathbf{0 . 0 7 2}$ \\
\hline SALE_VOL & $\mathbf{4 , 3 0 7}$ & $\mathbf{0 . 6 7 3}$ & $\mathbf{0 . 5 0 1}$ & $\mathbf{0 . 3 1 1}$ & $\mathbf{0 . 5 4 1}$ & $\mathbf{0 . 8 9 4}$ \\
\hline SPE_ITEM & $\mathbf{4 , 3 0 7}$ & $\mathbf{- 0 . 0 1 8}$ & $\mathbf{0 . 0 5 6}$ & $\mathbf{- 0 . 0 2 1}$ & $\mathbf{- 0 . 0 0 6}$ & $\mathbf{0 . 0 0 0}$ \\
\hline BIG4 & 4,307 & $\mathbf{0 . 9 2 8}$ & $\mathbf{0 . 2 5 8}$ & $\mathbf{1 . 0 0 0}$ & $\mathbf{1 . 0 0 0}$ & $\mathbf{1 . 0 0 0}$ \\
\hline HIGH_RISK & $\mathbf{4 , 3 0 7}$ & $\mathbf{0 . 2 3 6}$ & $\mathbf{0 . 4 2 5}$ & $\mathbf{0 . 0 0 0}$ & $\mathbf{0 . 0 0 0}$ & $\mathbf{0 . 0 0 0}$ \\
\hline
\end{tabular}


Main Results (H1 Testing)

\begin{tabular}{|c|c|c|c|c|c|c|c|c|}
\hline \multirow{4}{*}{$\begin{array}{l}\text { TABLE } 4 \text { - Panel A } \\
\text { Variable } \\
\end{array}$} & \multicolumn{8}{|c|}{ Dependent Variable $=$ LAWSUIT } \\
\hline & \multicolumn{8}{|c|}{ Logistic Regression } \\
\hline & \multicolumn{4}{|c|}{ Column 1} & \multicolumn{4}{|c|}{ Column 2} \\
\hline & Estimate & Chi-Square & $\mathrm{Pr}>\mathrm{ChiSq}$ & VIF & Estimate & Chi-Square & $\operatorname{Pr}>$ ChiSq & VIF \\
\hline Intercept & $-3.11 * * *$ & 213.95 & $<.0001$ & & $-2.71 * * *$ & 148.12 & $<.0001$ & \\
\hline CSR & $-0.04 * * *$ & 35.68 & $<.0001$ & 1.25 & & & & \\
\hline CSR_STRENGTH & & & & & $-0.02 * *$ & 4.53 & 0.033 & 1.57 \\
\hline CSR_CONCERN & & & & & $0.11 * * *$ & 72.53 & $<.0001$ & 1.70 \\
\hline SIZE & $0.25 * * *$ & 249.77 & $<.0001$ & 2.02 & $0.19 * * *$ & 98.66 & $<.0001$ & 2.78 \\
\hline MTB & 0.00 & 0.56 & 0.454 & 1.33 & 0.00 & 0.52 & 0.473 & 1.33 \\
\hline LEV & $-0.35 * * *$ & 7.31 & 0.007 & 1.61 & $-0.27 * *$ & 4.35 & 0.037 & 1.62 \\
\hline ROA & $1.05 * * *$ & 10.52 & 0.001 & 4.60 & $1.07 * * *$ & 10.83 & 0.001 & 4.60 \\
\hline CASHFL & -0.20 & 0.45 & 0.502 & 2.73 & -0.15 & 0.26 & 0.610 & 2.73 \\
\hline LOSS & $0.25 * * *$ & 17.56 & $<.0001$ & 1.93 & $0.23^{* * *}$ & 15.25 & $<.0001$ & 1.94 \\
\hline ZSCORE & $-0.02 * * *$ & 8.53 & 0.004 & 2.47 & $-0.02 * * *$ & 8.35 & 0.004 & 2.47 \\
\hline TOBINQ & $-0.10 * * *$ & 17.42 & $<.0001$ & 2.55 & $-0.11 * * *$ & 20.80 & $<.0001$ & 2.56 \\
\hline FIRMAGE & $0.07^{*}$ & 3.46 & 0.063 & 1.66 & 0.05 & 2.05 & 0.152 & 1.67 \\
\hline ASSETAGE & $-0.60 * * *$ & 17.04 & $<.0001$ & 1.53 & $-0.54 * * *$ & 13.62 & 0.000 & 1.54 \\
\hline MGR_ABLITY & 0.00 & 0.00 & 0.981 & 1.25 & -0.01 & 0.02 & 0.892 & 1.26 \\
\hline ACCRUAL & 0.41 & 1.21 & 0.272 & 1.35 & 0.40 & 1.18 & 0.278 & 1.35 \\
\hline SALE_VOL & $-0.12 * * *$ & 8.38 & 0.004 & 1.38 & $-0.11 * * *$ & 7.83 & 0.005 & 1.38 \\
\hline SPE_ITEM & $-2.08 * * *$ & 19.21 & $<.0001$ & 1.82 & $-2.05 * * *$ & 18.56 & $<.0001$ & 1.82 \\
\hline BIG4 & 0.09 & 1.78 & 0.182 & 1.16 & $0.13^{*}$ & 3.26 & 0.071 & 1.16 \\
\hline HIGH_RISK & 0.10 & 1.47 & 0.226 & 3.54 & 0.10 & 1.63 & 0.201 & 3.54 \\
\hline Industry Indicators & Yes & & & & Yes & & & \\
\hline Year Indicators & Yes & & & & Yes & & & \\
\hline Pseudo $\mathrm{R}^{2}$ & 0.1092 & & & & 0.1117 & & & \\
\hline Observations & 21,761 & & & & 21,761 & & & \\
\hline
\end{tabular}




\section{Main Results (H2 Testing)}

\begin{tabular}{|c|c|c|c|c|c|c|c|c|}
\hline Table 4 - Panel B & & & Depen & Varia & AWWUIT_ & & & \\
\hline & & & ustered & dard & s OLS Regr & & & \\
\hline & & Colum & & & & Colum & & \\
\hline Variable & Estimate & t Value & $\operatorname{Pr}>|t|$ & VIF & Estimate & t Value & $\operatorname{Pr}>|t|$ & VIF \\
\hline Intercept & -0.0007 & -0.21 & 0.833 & & -0.0019 & -0.51 & 0.613 & \\
\hline CSR & $0.0003 * * *$ & 3.33 & 0.001 & 1.36 & & & & \\
\hline CSR_STRENGTH & & & & & $0.0003 * *$ & 2.38 & 0.018 & 1.95 \\
\hline CSR_CONCERN & & & & & $-0.0005 * * *$ & -2.77 & 0.006 & 1.97 \\
\hline SIZE & $-0.0012 * * *$ & -4.31 & $<.0001$ & 2.02 & $-0.0010 * * *$ & -2.88 & 0.004 & 3.19 \\
\hline MTB & 0.0000 & 0.97 & 0.334 & 1.13 & 0.0000 & 0.98 & 0.329 & 1.13 \\
\hline LEV & $0.0059 * *$ & 2.31 & 0.021 & 1.64 & $0.0057 * *$ & 2.23 & 0.026 & 1.66 \\
\hline ROA & $-0.0605 * * *$ & -4.95 & $<.0001$ & 3.69 & $-0.0604 * * *$ & -4.94 & $<.0001$ & 3.69 \\
\hline CASHFL & $0.0498 * * *$ & 5.08 & $<.0001$ & 2.05 & $0.0495 * * *$ & 5.05 & $<.0001$ & 2.05 \\
\hline LOSS & -0.0022 & -1.48 & 0.139 & 1.88 & -0.0021 & -1.44 & 0.151 & 1.89 \\
\hline ZSCORE & $0.0003 * *$ & 2.04 & 0.042 & 2.40 & $0.0003 * *$ & 2.03 & 0.042 & 2.40 \\
\hline TOBINQ & $-0.0024 * * *$ & -4.41 & $<.0001$ & 2.29 & $-0.0023 * * *$ & -4.37 & $<.0001$ & 2.31 \\
\hline FIRMAGE & $0.0012 * *$ & 2.15 & 0.031 & 1.60 & $0.0013^{* *}$ & 2.21 & 0.027 & 1.61 \\
\hline ASSETAGE & 0.0045 & 1.58 & 0.115 & 1.54 & 0.0043 & 1.49 & 0.135 & 1.55 \\
\hline MGR_ABLITY & -0.0002 & -0.16 & 0.876 & 1.29 & -0.0002 & -0.17 & 0.863 & 1.29 \\
\hline ACCRUAL & -0.0113 & -1.05 & 0.295 & 1.30 & -0.0112 & -1.04 & 0.300 & 1.30 \\
\hline SALE_VOL & 0.0005 & 0.70 & 0.487 & 1.39 & 0.0005 & 0.70 & 0.486 & 1.39 \\
\hline SPE_ITEM & $0.2208 * * *$ & 9.49 & $<.0001$ & 1.84 & $0.2206^{* * *}$ & 9.48 & $<.0001$ & 1.84 \\
\hline BIG4 & 0.0018 & 1.22 & 0.223 & 1.15 & 0.0017 & 1.15 & 0.251 & 1.16 \\
\hline HIGH_RISK & 0.0006 & 0.43 & 0.669 & 3.72 & 0.0006 & 0.42 & 0.677 & 3.72 \\
\hline Industry Indicators & Yes & & & & Yes & & & \\
\hline Year Indicators & Yes & & & & Yes & & & \\
\hline Adjusted $\mathbf{R}^{2}$ & 0.2603 & & & & 0.2607 & & & \\
\hline Observations & 4,307 & & & & 4,307 & & & \\
\hline
\end{tabular}




\section{Additional Test \#1 - Alternative CSR Measures}

- Two alternative measures

1. Prior research including Kim et al. (2012) suggests excluding the corporate governance and the human rights components from the net CSR score because the former focuses on a firm's governance mechanism and the latter does not have sufficient data.

CSR_ALT $=($ Total strengths of Community Relations - Total concerns of Community Relations) + (Total strengths of Diversity - Total concerns of Diversity) + (Total strengths of Employee Relations - Total concerns of Employee Relations) + (Total strengths of Environment - Total concerns of Environment $)+($ Total strengths of Product - Total concerns of Product)

2. H_CSR which equals one if an observation's net CSR score (CSR) is above the median and zero otherwise. 


\section{Additional Test \#1 - Alternative CSR Measures}

\begin{tabular}{|c|c|c|c|c|c|c|c|c|c|c|c|c|}
\hline Table 5 & & \multicolumn{5}{|c|}{ Dependent Variable = LAWSUIT } & \multicolumn{6}{|c|}{ Dependent Variable = LAWSUIT_GL } \\
\hline & \multicolumn{6}{|c|}{ Logistic Regression } & \multicolumn{6}{|c|}{ Clustered Standard Errors OLS } \\
\hline & \multicolumn{3}{|c|}{ Column 1} & \multicolumn{3}{|c|}{ Column 2} & \multicolumn{3}{|c|}{ Column 3} & \multicolumn{3}{|c|}{ Column 4} \\
\hline Variable & Estimate & Chi-Square & $\operatorname{Pr}>$ ChiSq & Estimate & Chi-Square & $\operatorname{Pr}>$ ChiSq & Estimate & t Value & $\operatorname{Pr}>|t|$ & Estimate & t Value & $\operatorname{Pr}>|t|$ \\
\hline Intercept & $-3.13 * * *$ & 214.58 & $<.0001$ & $-3.01 * * *$ & 202.56 & $<.0001$ & -0.0003 & -0.08 & 0.936 & -0.0014 & -0.40 & 0.690 \\
\hline CSR_ALT & $-0.04 * * *$ & 23.57 & $<.0001$ & & & & $0.0004 * * *$ & 3.26 & 0.001 & & & \\
\hline H_CSR & & & & $-0.18 * * *$ & 17.73 & $<.0001$ & & & & $0.0015 * *$ & 2.50 & 0.012 \\
\hline МТВ & 0.00 & 0.54 & 0.461 & 0.00 & 0.49 & 0.485 & 0.0000 & 0.95 & 0.342 & 0.0000 & 0.98 & 0.325 \\
\hline LEV & $-0.35 * * *$ & 7.27 & 0.007 & $-0.34 * * *$ & 7.02 & 0.008 & $0.0059 * *$ & 2.32 & 0.020 & $0.0060 * *$ & 2.35 & 0.019 \\
\hline ROA & $1.06^{* * *}$ & 10.79 & 0.001 & $1.09^{* * *}$ & 11.25 & 0.001 & $-0.0605 * * *$ & -4.96 & $<.0001$ & $-0.0609 * * *$ & -4.99 & $<.0001$ \\
\hline CASHFL & -0.21 & 0.52 & 0.472 & -0.27 & 0.81 & 0.367 & $0.0498 * * *$ & 5.08 & $<.0001$ & $0.0504 * * *$ & 5.15 & $<.0001$ \\
\hline LOSS & $0.25 * * *$ & 17.63 & $<.0001$ & $0.25 * * *$ & 17.95 & $<.0001$ & -0.0022 & -1.49 & 0.137 & -0.0022 & -1.53 & 0.126 \\
\hline ZSCORE & $-0.02 * * *$ & 8.77 & 0.003 & $-0.02 * * *$ & 8.57 & 0.003 & $0.0003 * *$ & 2.05 & 0.040 & $0.0003 * *$ & 2.05 & 0.041 \\
\hline TOBINQ & $-0.10 * * *$ & 17.27 & $<.0001$ & $-0.10 * * *$ & 17.57 & $<.0001$ & $-0.0024 * * *$ & -4.44 & $<.0001$ & $-0.0024 * * *$ & -4.41 & $<.0001$ \\
\hline ASSETAGE & $-0.60 * * *$ & 17.23 & $<.0001$ & $-0.58 * * *$ & 16.09 & $<.0001$ & 0.0046 & 1.60 & 0.109 & 0.0043 & 1.51 & 0.132 \\
\hline MGR_ABLITY & 0.00 & 0.00 & 0.965 & -0.01 & 0.01 & 0.926 & -0.0002 & -0.17 & 0.862 & -0.0001 & -0.09 & 0.927 \\
\hline ACCRUAL & 0.41 & 1.19 & 0.274 & 0.38 & 1.03 & 0.309 & -0.0114 & -1.05 & 0.293 & -0.0111 & -1.03 & 0.304 \\
\hline SALE_VOL & $-0.11 * * *$ & 8.02 & 0.005 & $-0.11 * * *$ & 7.86 & 0.005 & 0.0005 & 0.68 & 0.495 & 0.0005 & 0.65 & 0.514 \\
\hline SPE_ITEM & $-2.10 * * *$ & 19.67 & $<.0001$ & $-2.12 * * *$ & 19.98 & $<.0001$ & $0.2210^{* * *}$ & 9.50 & $<.0001$ & $0.2216^{* * *}$ & 9.51 & $<.0001$ \\
\hline BIG4 & 0.09 & 1.79 & 0.181 & 0.10 & 1.96 & 0.161 & 0.0018 & 1.22 & 0.224 & 0.0018 & 1.19 & 0.235 \\
\hline HIGH_RISK & 0.09 & 1.40 & 0.237 & 0.08 & 1.14 & 0.285 & 0.0006 & 0.41 & 0.683 & 0.0007 & 0.52 & 0.606 \\
\hline Industry Indicators & Yes & & & Yes & & & Yes & & & Yes & & \\
\hline Year Indicators & Yes & & & Yes & & & Yes & & & Yes & & \\
\hline Pseudo $R^{2} /$ Adj. $R^{2}$ & 0.1084 & & & 0.1080 & & & 0.2602 & & & 0.2596 & & \\
\hline Observations & 21,761 & & & 21,761 & & & 4,307 & & & 4,307 & & \\
\hline
\end{tabular}




\section{Additional Test \#2 - Alternative Sample Periods}

\begin{tabular}{|c|c|c|c|c|c|c|c|c|c|c|c|c|}
\hline Table 6 & & \multicolumn{5}{|c|}{ Dependent Variable = LAWSUIT } & \multicolumn{6}{|c|}{ Dependent Variable = LAWSUIT_GL } \\
\hline & \multicolumn{6}{|c|}{ Logistic Regression } & \multicolumn{6}{|c|}{ Clustered Standard Errors OLS } \\
\hline & \multicolumn{3}{|c|}{ Column 1} & \multicolumn{3}{|c|}{ Column 2} & \multicolumn{3}{|c|}{ Column 3} & \multicolumn{3}{|c|}{ Column 4} \\
\hline & \multicolumn{3}{|c|}{ 1991-2002 } & \multicolumn{3}{|c|}{ 2003-2016 } & \multicolumn{3}{|c|}{ 1991-2002 } & \multicolumn{3}{|c|}{ 2003-2016 } \\
\hline Variable & Estimate & Chi-Square & $\operatorname{Pr}>$ ChiSq & Estimate & Chi-Square & $\operatorname{Pr}>$ ChiSq & Estimate & t Value & $\operatorname{Pr}>|t|$ & Estimate & t Value & $\operatorname{Pr}>|t|$ \\
\hline Intercept & $-3.85 * * *$ & 16.67 & $<.0001$ & $-3.03 * * *$ & 221.59 & $<.0001$ & $-0.0390 * *$ & -2.06 & 0.041 & 0.0027 & 0.75 & 0.451 \\
\hline CSR & $-0.04 *$ & 3.35 & 0.067 & $-0.05 * * *$ & 39.01 & $<.0001$ & 0.0004 & 0.66 & 0.513 & $0.0003 * * *$ & 3.13 & 0.002 \\
\hline SIZE & $0.47 * * *$ & 50.77 & $<.0001$ & $0.25 * * *$ & 232.26 & $<.0001$ & -0.0001 & -0.07 & 0.940 & $-0.0014 * * *$ & -4.63 & $<.0001$ \\
\hline MTB & 0.01 & 0.46 & 0.498 & 0.00 & 0.51 & 0.476 & $0.0001 * *$ & 2.02 & 0.045 & 0.0000 & 0.44 & 0.659 \\
\hline LEV & 0.03 & 0.00 & 0.963 & $-0.34 * * *$ & 7.02 & 0.008 & 0.0046 & 0.26 & 0.797 & $0.0051 * *$ & 1.97 & 0.048 \\
\hline ROA & 0.04 & 0.00 & 0.975 & $0.98 * * *$ & 8.74 & 0.003 & $-0.1133 * * *$ & -2.83 & 0.005 & $-0.0486 * * *$ & -4.02 & $<.0001$ \\
\hline CASHFL & $-3.96 * * *$ & 8.60 & 0.003 & -0.11 & 0.14 & 0.710 & $0.1079 * * *$ & 3.00 & 0.003 & $0.0452 * * *$ & 4.57 & $<.0001$ \\
\hline LOSS & 0.29 & 1.93 & 0.165 & $0.24 * * *$ & 15.34 & $<.0001$ & $-0.0105^{*}$ & -1.72 & 0.086 & -0.0009 & -0.56 & 0.576 \\
\hline ZSCORE & 0.03 & 2.09 & 0.148 & $-0.02 * * *$ & 9.55 & 0.002 & 0.0008 & 1.30 & 0.194 & 0.0002 & 0.85 & 0.398 \\
\hline TOBINQ & 0.00 & 0.00 & 0.973 & $-0.10 * * *$ & 17.48 & $<.0001$ & -0.0032 & -1.04 & 0.298 & $-0.0025 * * *$ & -4.65 & $<.0001$ \\
\hline FIRMAGE & $-0.35 * *$ & 5.29 & 0.022 & 0.06 & 2.52 & 0.112 & 0.0052 & 1.64 & 0.103 & $0.0012 * *$ & 2.17 & 0.030 \\
\hline ASSETAGE & $-2.13 * * *$ & 8.04 & 0.005 & $-0.58 * * *$ & 15.39 & $<.0001$ & 0.0351 & 1.59 & 0.113 & 0.0038 & 1.34 & 0.181 \\
\hline MGR_ABLITY & $-0.51 * *$ & 4.54 & 0.033 & 0.00 & 0.00 & 0.945 & -0.0084 & -1.58 & 0.116 & 0.0006 & 0.49 & 0.621 \\
\hline ACCRUAL & $2.64 *$ & 2.78 & 0.095 & 0.33 & 0.76 & 0.383 & 0.0052 & 0.14 & 0.891 & -0.0112 & -1.03 & 0.305 \\
\hline SALE_VOL & -0.27 & 2.42 & 0.120 & $-0.11 * * *$ & 7.51 & 0.006 & 0.0030 & 0.65 & 0.514 & 0.0005 & 0.61 & 0.539 \\
\hline SPE_ITEM & $-3.72 * *$ & 4.24 & 0.040 & $-1.45 * * *$ & 8.75 & 0.003 & $0.2069 * * *$ & 2.85 & 0.005 & $0.2178 * * *$ & 8.66 & $<.0001$ \\
\hline BIG4 & -0.25 & 0.24 & 0.624 & $0.12^{*}$ & 3.05 & 0.081 & -0.0070 & -0.99 & 0.323 & 0.0019 & 1.26 & 0.209 \\
\hline HIGH_RISK & -0.10 & 0.07 & 0.795 & 0.10 & 1.49 & 0.222 & -0.0078 & -0.87 & 0.386 & 0.0008 & 0.56 & 0.578 \\
\hline Industry Indicators & Yes & & & Yes & & & Yes & & & Yes & & \\
\hline Year Indicators & Yes & & & Yes & & & Yes & & & Yes & & \\
\hline Pseudo $\mathbf{R}^{2}$ / Adj. $\mathbf{R}^{2}$ & 0.1386 & & & 0.0710 & & & 0.2968 & & & 0.2532 & & \\
\hline Observations & 3,131 & & & 18,630 & & & 279 & & & 4,028 & & \\
\hline
\end{tabular}




\section{Additional Test \#3 - Lagged CSR Measures in H1}

\begin{tabular}{|c|c|c|c|c|c|c|c|c|c|}
\hline \multirow[t]{2}{*}{ Table 7} & & \multicolumn{8}{|c|}{ Dependent Variable = LAWSUIT } \\
\hline & \multicolumn{9}{|c|}{ Logistic Regression } \\
\hline & \multicolumn{3}{|c|}{ Column 1} & \multicolumn{3}{|c|}{ Column 2} & \multicolumn{3}{|c|}{ Column 3} \\
\hline Variable & Estimate & Chi-Square & $\operatorname{Pr}>$ ChiSq & Estimate & Chi-Square & $\operatorname{Pr}>$ ChiSq & Estimate & Chi-Square & $\mathrm{Pr}>\mathrm{ChiSq}$ \\
\hline LAG_CSR1 & $-0.04 * * *$ & 17.35 & $<.0001$ & & & & & & \\
\hline LAG_CSR2 & & & & $-0.04 * * *$ & 17.99 & $<.0001$ & & & \\
\hline LAG_CSR3 & & & & & & & $-0.03 * * *$ & 15.18 & $<.0001$ \\
\hline MTB & $0.01 *$ & 3.13 & 0.077 & $0.01 *$ & 3.43 & 0.064 & $0.01 *$ & 2.90 & 0.089 \\
\hline LEV & $-0.33 * *$ & 4.36 & 0.037 & $-0.36 * *$ & 4.40 & 0.036 & $-0.55 * * *$ & 9.31 & 0.002 \\
\hline ROA & $1.25 * *$ & 6.03 & 0.014 & $1.20 * *$ & 4.89 & 0.027 & $1.30 * *$ & 5.15 & 0.023 \\
\hline CASHFL & $-0.73 *$ & 2.83 & 0.093 & -0.75 & 2.67 & 0.102 & -0.66 & 1.86 & 0.173 \\
\hline LOSS & $0.18 * *$ & 6.08 & 0.014 & $0.21 * * *$ & 7.16 & 0.007 & $0.24 * * *$ & 8.22 & 0.004 \\
\hline ZSCORE & $-0.02 * * *$ & 6.75 & 0.009 & $-0.03 * * *$ & 7.25 & 0.007 & $-0.04 * * *$ & 13.62 & 0.000 \\
\hline TOBINQ & $-0.14 * * *$ & 16.60 & $<.0001$ & $-0.12 * * *$ & 12.86 & 0.000 & $-0.12 * * *$ & 11.09 & 0.001 \\
\hline$\overline{\text { ASSETAGE }}$ & $-0.40 * *$ & 5.16 & 0.023 & $-0.39 * *$ & 4.40 & 0.036 & $-0.37 *$ & 3.54 & 0.060 \\
\hline MGR_ABLITY & 0.02 & 0.04 & 0.843 & -0.01 & 0.01 & 0.925 & -0.01 & 0.02 & 0.901 \\
\hline ACCRUAL & $1.32 * *$ & 6.48 & 0.011 & $1.36 * *$ & 6.10 & 0.014 & $1.20 * *$ & 4.20 & 0.041 \\
\hline SALE_VOL & $-0.13 * *$ & 6.60 & 0.010 & $-0.16 * * *$ & 8.12 & 0.004 & $-0.14 * *$ & 5.47 & 0.019 \\
\hline SPE_ITEM & $-2.91 * * *$ & 17.83 & $<.0001$ & $-2.77 * * *$ & 14.29 & 0.000 & $-2.36 * * *$ & 9.02 & 0.003 \\
\hline BIG4 & 0.14 & 2.62 & 0.106 & 0.14 & 2.54 & 0.111 & 0.12 & 1.64 & 0.200 \\
\hline HIGH_RISK & 0.02 & 0.04 & 0.847 & 0.05 & 0.23 & 0.633 & 0.05 & 0.28 & 0.600 \\
\hline Industry Indicators & Yes & & & Yes & & & Yes & & \\
\hline Year Indicators & Yes & & & Yes & & & Yes & & \\
\hline Pseudo $\mathbf{R}^{2}$ & 0.1044 & & & 0.1020 & & & 0.0976 & & \\
\hline Observations & 16,099 & & & 14,452 & & & 12,915 & & \\
\hline
\end{tabular}




\section{Additional Test \#4 - Change Analysis}

\begin{tabular}{|l|}
\hline Table 8 \\
\hline \\
\hline Variable \\
\hline Intercept \\
\hline$\Delta$ CSR \\
\hline$\Delta$ SIZE \\
\hline$\Delta$ MTB \\
\hline$\Delta$ LEV \\
\hline$\Delta$ ROA \\
\hline$\Delta$ CASHFL \\
\hline$\Delta$ LOSS \\
\hline$\triangle$ ZSCORE \\
\hline$\triangle$ TOBINQ \\
\hline$\Delta$ FIRMAGE \\
\hline$\triangle$ ASSETAGE \\
\hline$\Delta$ MGR_ABLITY \\
\hline$\triangle$ ACCRUAL \\
\hline$\triangle$ SALE_VOL \\
\hline$\triangle$ SPE_ITEM \\
\hline$\triangle$ BIG4 \\
\hline Industry Indicators \\
\hline Year Indicators \\
\hline Adj. R \\
\hline Observations \\
\hline
\end{tabular}

\begin{tabular}{|c|c|c|c|c|c|}
\hline & Column 1 & & & Column 2 & \\
\hline \multicolumn{3}{|c|}{ Dependent Variable $=\Delta$ LAWSUIT } & \multicolumn{3}{|c|}{ Dependent Variable $=\Delta$ LAWSUIT_GL } \\
\hline & Full Sample & & & Lawsuit Sample & \\
\hline Estimate & t Value & $\operatorname{Pr}>|t|$ & Estimate & t Value & $\operatorname{Pr}>|t|$ \\
\hline-0.003 & -0.13 & 0.894 & 0.0019 & 1.01 & 0.312 \\
\hline$-0.006 * *$ & -2.41 & 0.016 & $0.0001 *$ & 1.73 & 0.083 \\
\hline $0.048^{*}$ & 1.75 & 0.081 & $-0.0044 *$ & -1.84 & 0.066 \\
\hline 0.000 & -0.04 & 0.971 & $0.0001 *$ & 1.92 & 0.056 \\
\hline-0.040 & -0.56 & 0.573 & $0.0149 * *$ & 2.29 & 0.022 \\
\hline $0.231 * *$ & 2.44 & 0.015 & $-0.0506 * * *$ & -3.11 & 0.002 \\
\hline-0.029 & -0.37 & 0.708 & $0.0328^{* * *}$ & 2.91 & 0.004 \\
\hline 0.012 & 0.98 & 0.329 & 0.0007 & 0.37 & 0.711 \\
\hline$-0.007 * *$ & -2.29 & 0.022 & 0.0007 & 1.61 & 0.107 \\
\hline 0.009 & 0.90 & 0.366 & $-0.0038 * * *$ & -2.73 & 0.006 \\
\hline-0.013 & -0.09 & 0.925 & 0.0048 & 0.91 & 0.361 \\
\hline-0.165 & -1.58 & 0.113 & $0.0162 *$ & 1.85 & 0.064 \\
\hline-0.023 & -1.29 & 0.197 & -0.0009 & -0.64 & 0.522 \\
\hline $0.203 * *$ & 2.29 & 0.022 & -0.0066 & -0.58 & 0.564 \\
\hline-0.004 & -0.22 & 0.828 & 0.0002 & 0.12 & 0.902 \\
\hline$-0.347 * * *$ & -2.77 & 0.006 & $0.2282 * * *$ & 8.92 & $<.0001$ \\
\hline 0.027 & 0.62 & 0.536 & -0.0008 & -0.20 & 0.844 \\
\hline Yes & & & Yes & & \\
\hline Yes & & & Yes & & \\
\hline 0.0044 & & & 0.2235 & & \\
\hline 16,099 & & & 2,465 & & \\
\hline
\end{tabular}




\section{Additional Test \#5 - Two-Stage OLS Regression Analysis H2}

\begin{tabular}{|l|}
\hline Table 9 \\
\hline \\
\hline \\
\hline Variable \\
\hline Intercept \\
\hline CSR_Mean \\
\hline CSR_Instrumental \\
\hline SIZE \\
\hline MTB \\
\hline LEV \\
\hline ROA \\
\hline CASHFL \\
\hline LOSS \\
\hline ZSCORE \\
\hline TOBINQ \\
\hline FIRMAGE \\
\hline ASSETAGE \\
\hline MGR_ABLITY \\
\hline ACCRUAL \\
\hline SALE_VOL \\
\hline SPE_ITEM \\
\hline BIG4 \\
\hline HIGH_RISK \\
\hline Industry Indicators \\
\hline Year Indicators \\
\hline Adj. R \\
\hline Observations \\
\hline \\
\hline
\end{tabular}

\begin{tabular}{|c|c|c|c|c|c|}
\hline \multicolumn{6}{|c|}{ Dependent Variable $=$ LAWSUIT_GL } \\
\hline \multicolumn{6}{|c|}{ Lawsuit Sample } \\
\hline \multicolumn{6}{|c|}{ Ordinary Least Squares Regression (OLS) } \\
\hline \multicolumn{3}{|c|}{ Column 1} & \multicolumn{3}{|c|}{ Column 2} \\
\hline & Stage 1 & & & Stage 2 & \\
\hline Estimate & t Value & $\operatorname{Pr}>|t|$ & Estimate & t Value & $\operatorname{Pr}>|t|$ \\
\hline$-3.048 * * *$ & -6.67 & $<.0001$ & -0.0007 & -0.21 & 0.831 \\
\hline \multirow{2}{*}{$0.885 * * *$} & 9.35 & $<.0001$ & & & \\
\hline & & & $0.0003 * * *$ & 2.98 & 0.003 \\
\hline $0.470 * * *$ & 14.11 & $<.0001$ & $-0.0012 * * *$ & -4.64 & $<.0001$ \\
\hline 0.008 & 1.40 & 0.160 & 0.0000 & 0.91 & 0.362 \\
\hline-0.326 & -1.21 & 0.228 & $0.0059 * * *$ & 2.90 & 0.004 \\
\hline$-1.907 * * *$ & -2.86 & 0.004 & $-0.0605 * * *$ & -12.02 & $<.0001$ \\
\hline $2.151 * * *$ & 3.26 & 0.001 & $0.0498 * * *$ & 10.02 & $<.0001$ \\
\hline$-0.290 * *$ & -2.32 & 0.021 & $-0.0022 * *$ & -2.30 & 0.022 \\
\hline 0.002 & 0.12 & 0.904 & $0.0003 * * *$ & 3.10 & 0.002 \\
\hline $0.181 * * *$ & 3.59 & 0.000 & $-0.0024 * * *$ & -6.19 & $<.0001$ \\
\hline $0.195 * *$ & 2.59 & 0.010 & $0.0012 * *$ & 2.13 & 0.033 \\
\hline$-1.042 * * *$ & -3.31 & 0.001 & $0.0045^{*}$ & 1.89 & 0.059 \\
\hline $0.528 * * *$ & 3.69 & 0.000 & -0.0002 & -0.16 & 0.875 \\
\hline-0.029 & -0.04 & 0.971 & $-0.0113^{*}$ & -1.83 & 0.067 \\
\hline$-0.236 * * *$ & -2.68 & 0.007 & 0.0005 & 0.83 & 0.409 \\
\hline $1.751^{*}$ & 1.92 & 0.055 & $0.2208 * * *$ & 32.12 & $<.0001$ \\
\hline-0.162 & -1.04 & 0.299 & 0.0018 & 1.56 & 0.119 \\
\hline $0.306^{*}$ & 1.80 & 0.072 & 0.0006 & 0.45 & 0.650 \\
\hline Yes & & & Yes & & \\
\hline Yes & & & Yes & & \\
\hline 0.2817 & & & 0.2603 & & \\
\hline 4,307 & & & 4,307 & & \\
\hline
\end{tabular}




\section{Additional Test \#6 - Settlement Gain vs. Settlement Loss in H2}

\begin{tabular}{|l|l|}
\hline Table 10 \\
\hline \\
\hline \\
\hline Variable \\
\hline Intercept \\
\hline CSR \\
\hline SIZE \\
\hline MTB \\
\hline LEV \\
\hline ROA \\
\hline CASHFL \\
\hline LOSS \\
\hline ZSCORE \\
\hline TOBINQ \\
\hline FIRMAGE \\
\hline ASSETAGE \\
\hline MGR_ABLITY \\
\hline ACCRUAL \\
\hline SALE_VOL \\
\hline SPE_ITEM \\
\hline BIG4 \\
\hline HIGH_RISK \\
\hline Industry Indicators \\
\hline Year Indicators \\
\hline Adj. R \\
\hline Observations \\
\hline
\end{tabular}

\section{Dependent Variable $=$ LAWSUIT_GL \\ Lawsuit Sample \\ Clustered Standard Errors OLS Regression \\ Column 1} Settlement Gain

Estimate

0.000

$-0.0021 * * *$

$-0.0022-0.80$

\begin{tabular}{l|l}
$-0.0473 * *$ & -2.58
\end{tabular}

\begin{tabular}{l|l}
$0.0424 * * *$ & 3.01
\end{tabular}

\begin{tabular}{l|l}
$0.0037 * *$ & 2.13 \\
\hline
\end{tabular}

\begin{tabular}{ll}
-0.0003 & -1.42 \\
\hline
\end{tabular}

\begin{tabular}{l|l}
0.0000 & -0.07
\end{tabular}

\begin{tabular}{l|l}
0.0010 & 1.34 \\
\hline
\end{tabular}

\begin{tabular}{l|l}
0.0012 & 0.33
\end{tabular}

$0.0021 \quad 1.60$

\begin{tabular}{l|l}
0.0179 & 1.26
\end{tabular}

\begin{tabular}{l|l}
$0.0021 * *$ & 2.16
\end{tabular}

\begin{tabular}{l|l}
$0.1798 * * *$ & 5.22
\end{tabular}

\begin{tabular}{l|l}
-0.0002 & -0.10 \\
\hline
\end{tabular}

\begin{tabular}{l|l}
0.0012 & 0.66
\end{tabular}

Yes

Yes

0.2876

2,050
Column 2

Settlement Loss

\begin{tabular}{|c|c|c|c|}
\hline & \multicolumn{3}{|c|}{ Settlement Loss } \\
\hline Pr $>|t|$ & Estimate & $t$ Value & $\operatorname{Pr}>|t|$ \\
\hline 0.024 & $-0.0168 * * *$ & -3.92 & $\mathbf{0}$ \\
\hline 0.456 & $\mathbf{0 . 0 0 0 3 * * *}$ & 2.72 & 0.007 \\
\hline$<.0001$ & $0.0009 * *$ & 2.47 & 0.014 \\
\hline 0.172 & 0.0001 & 0.92 & 0.001 \\
\hline 0.425 & $0.0110 * * *$ & 3.21 & $<.000$ \\
\hline 0.010 & $-0.0486 * * *$ & -3.98 & 0.001 \\
\hline 0.003 & $0.0352 * * *$ & 3.37 & 0.001 \\
\hline 0.033 & $-0.0062 * * *$ & -3.39 & 0.048 \\
\hline 0.157 & $0.0004 * *$ & 1.98 & $<.000$ \\
\hline 0.948 & $-0.0030 * * *$ & -4.13 & 0.239 \\
\hline 0.181 & 0.0008 & 1.18 & 0.027 \\
\hline 0.739 & $0.0082 * *$ & 2.21 & 0.582 \\
\hline 0.111 & -0.0007 & -0.55 & 0.004 \\
\hline 0.208 & $-0.0371 * * *$ & -2.89 & 0.315 \\
\hline 0.031 & 0.0010 & 1.00 & $<.000$ \\
\hline$<.0001$ & $0.1617 * * *$ & 6.60 & 0.156 \\
\hline 0.922 & 0.0022 & 1.42 & 0.858 \\
\hline 0.513 & 0.0003 & 0.18 & \\
\hline
\end{tabular}

Yes

0.3122

2,257 


\begin{tabular}{|c|c|c|c|c|c|c|}
\hline \multirow[t]{4}{*}{ Table 11} & \multicolumn{6}{|c|}{ Dependent Variable $=$ LAWSUIT } \\
\hline & \multicolumn{6}{|c|}{ Full Sample } \\
\hline & \multicolumn{6}{|c|}{ Logistic Regression } \\
\hline & \multicolumn{3}{|c|}{ Column 1} & \multicolumn{3}{|c|}{ Column 2} \\
\hline & \multicolumn{3}{|c|}{ High Cash Holdings } & \multicolumn{3}{|c|}{ Low Cash Holdings } \\
\hline Variable & Estimate & Chi-Square & $\operatorname{Pr}>$ ChiSq & Estimate & Chi-Square & $\operatorname{Pr}>\mathrm{ChiSq} \mid$ \\
\hline Intercept & $-2.622 * * *$ & 79.69 & $<.0001$ & $-3.434 * * *$ & 85.90 & $<.0001$ \\
\hline CSR & $-0.037 * * *$ & 17.24 & $<.0001$ & $-0.066 * * *$ & 14.64 & 0.000 \\
\hline SIZE & $0.205^{* * *}$ & 71.63 & $<.0001$ & $0.248 * * *$ & 53.00 & $<.0001$ \\
\hline MTB & 0.008 & 2.22 & 0.136 & -0.008 & 1.13 & 0.287 \\
\hline LEV & -0.256 & 1.89 & 0.169 & $-0.423 * *$ & 5.03 & 0.025 \\
\hline ROA & $1.543 * * *$ & 9.46 & 0.002 & $0.796^{*}$ & 3.29 & 0.070 \\
\hline CASHFL & $-0.827^{*}$ & 3.38 & 0.066 & 0.062 & 0.02 & 0.880 \\
\hline LOSS & 0.112 & 1.68 & 0.196 & $0.378^{* * *}$ & 20.20 & $<.0001$ \\
\hline ZSCORE & $-0.021 * *$ & 5.23 & 0.022 & $-0.021 * *$ & 4.76 & 0.029 \\
\hline TOBINQ & $-0.127 * * *$ & 15.16 & $<.0001$ & -0.055 & 2.42 & 0.120 \\
\hline FIRMAGE & 0.036 & 0.53 & 0.465 & $0.153 * * *$ & 7.76 & 0.005 \\
\hline ASSETAGE & $-0.580 * * *$ & 8.06 & 0.005 & $-0.494 * *$ & 5.19 & 0.023 \\
\hline MGR_ABLITY & 0.019 & 0.05 & 0.830 & 0.020 & 0.03 & 0.858 \\
\hline ACCRUAL & $1.362 * * *$ & 6.75 & 0.009 & -0.566 & 1.05 & 0.305 \\
\hline SALE_VOL & $-0.218 * * *$ & 14.66 & 0.000 & -0.005 & 0.01 & 0.936 \\
\hline SPE_ITEM & $-3.403 * * *$ & 21.67 & $<.0001$ & -1.072 & 2.70 & 0.101 \\
\hline BIG4 & 0.086 & 0.46 & 0.496 & 0.136 & 2.50 & 0.114 \\
\hline HIGH_RISK & $0.184^{*}$ & 3.17 & 0.075 & -0.031 & 0.06 & 0.806 \\
\hline Industry Indicators & Yes & & & Yes & & \\
\hline Year Indicators & Yes & & & Yes & & \\
\hline Pseudo $\mathbf{R}^{2}$ & 0.1193 & & & 0.1007 & & \\
\hline \multirow[t]{3}{*}{ Observations } & 10,881 & & & 10,880 & & \\
\hline & \multicolumn{6}{|c|}{ Coefficient Comparison Test } \\
\hline & \multicolumn{6}{|c|}{$\begin{array}{l}\text { Coefficient of CSR (-0.037) for Observations with High Cash Holdings vs. Coefficient of CSR }(-0.066) \text { for Observations with Low Cash } \\
\text { Holdings }\end{array}$} \\
\hline & & & F-stat. $=1$ & alue $<0.000$ & & 30 \\
\hline
\end{tabular}




\section{Additional Test \#8 - Using Individual CSR Components}

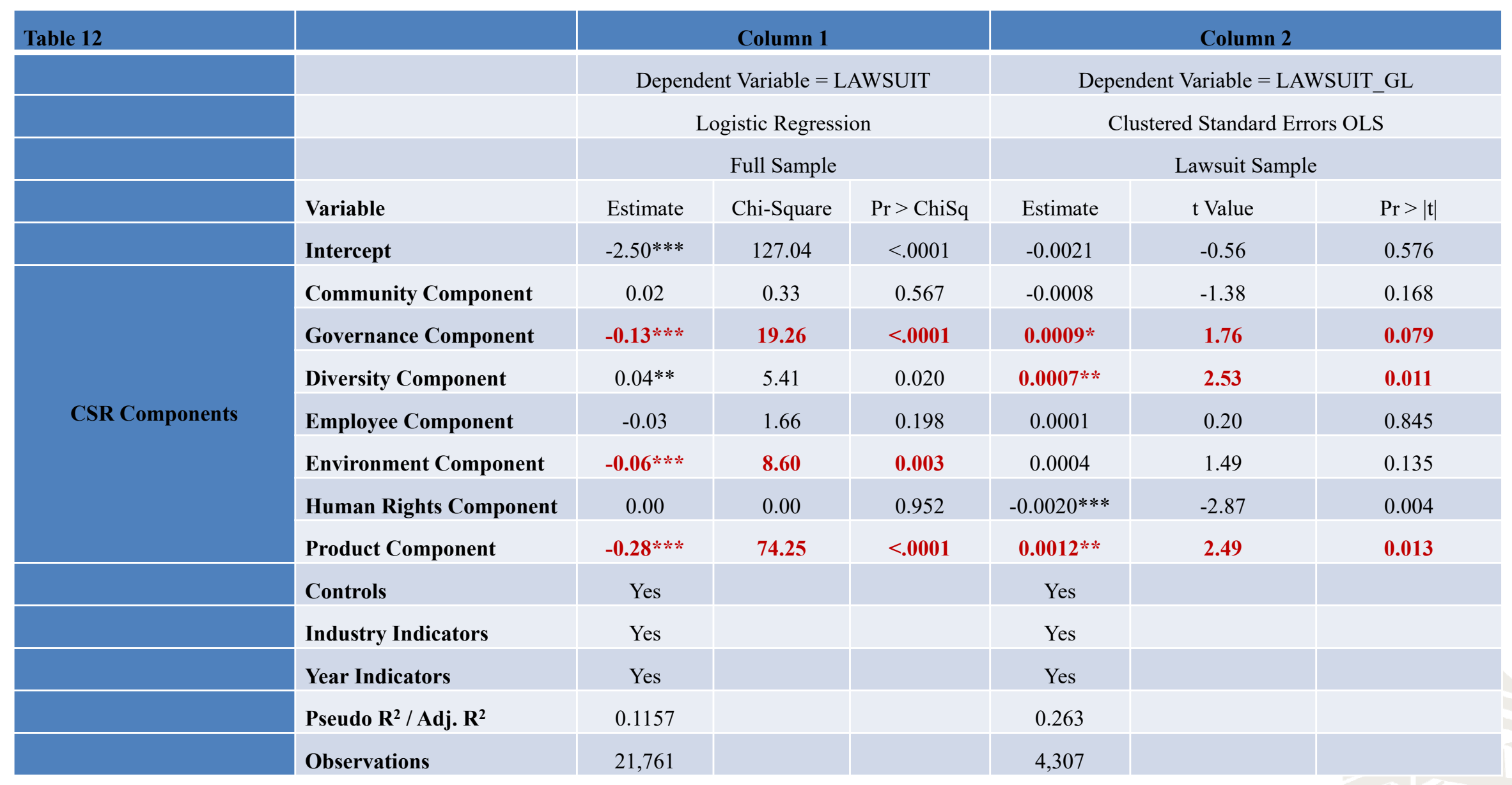




\section{Conclusion}

- In this study, we find that socially responsible firms are less likely to be involved in lawsuits. We also find that socially responsible firms receive favorable settlement outcomes (i.e., larger settlement gain or smaller settlement loss).

- Results are consistent with the stakeholder view of CSR.

- Several shortcomings: (1) large sample firms, (2) the lack of data on the nature of lawsuits, (3) other CSR theories exist. 


\section{Thank You}

huntsman.usu.edu 